Gazi University
Journal of Science
http://dergipark.gov.tr/gujs

\title{
The Use of Artificial Neural Networks Optimized with Fire Fly Algorithm in Cancer Diagnosis
}

\author{
Mehmet BILEN ${ }^{1, *}$ (D) Ali Hakan ISIK $^{2}$, Tuncay YIGIT ${ }^{3}$ \\ ${ }^{1}$ Mehmet Akif Ersoy University, Cavdir Vocational High School, 15000 Burdur, Turkey \\ ${ }^{2}$ Mehmet Akif Ersoy University, Faculty of Engineering and Architecture, 15000, Burdur, Turkey \\ ${ }^{3}$ Süleyman Demirel University, Faculty of Engineering, 32000, Isparta, Turkey
}

\section{Highlights}

- This paper focuses on classification process for central nervous system tumor.

- A hybrid approach is proposed for classification Microarray Data in the study.

- A highly precise and more efficient classification accuracy were obtained.

\section{Article Info}

Received: $17 / 10 / 2018$

Accepted: 05/06/2019

\section{Keywords}

Cancer Diagnosis Firefly Algorithm Hybrid Algorithms Artificial Neural Networks

\begin{abstract}
Today, the amount of biological data types obtained are increasing every day. Among these data types are micro arrays that play an important role in cancer diagnosis. The data analysis that are carried out through traditional approaches have proven unsuccessful in delivering efficient results on data types where data complexity is high and where sampling is low. For this reason, using a hybrid algorithm by merging the effective features of two distinct algorithms will yield effective results. In this study, a classification process was performed firstly by dimension reduction on micro array data that were obtained from the tissues from patients with a tumor in their central nervous system and then by using an artificial neural network algorithm that was optimized through Fire Fly Algorithm (FF), a hybrid approach. The data obtained were compared to K Nearest Neighbors (KNN), Support Vector Machine (SVM) and Artificial Neural Networks (ANN) classification algorithms, which are frequently used in the literature. Also, the results were compared to the findings that were obtained from artificial neural networks, which are reinforced by Genetic Algorithm (GA), another hybrid approach. Then the results were shared. The performance results obtained show that hybrid approaches present a highly precise and more efficient classification process but they show a slower performance than basic classification algorithms.
\end{abstract}

\section{INTRODUCTION}

Every day, increasing biological data amount brings about problems in the analysis of these data. Through traditional approaches, the analysis and the classification of these data types where data size and complexity is too much is too difficult and takes a lot of time. Micro array data are of great importance in cancer diagnosis [1]. Thanks to microarray technology, analysis can be done on thousands of gene expression at the same time. However, it is difficult to analyze by traditional approaches such as statistical methods because of its high complexity and large size. Heuristic algorithms are used frequently for the interpretation of these data [2-7]. However, due to the deficiencies, heuristic algorithms that can solve complex problems effectively may not always reach to the solution. Artificial Neural Networks, a powerful heuristic approach, are widely used in the classification problems on micro array data for cancer diagnosis [8-11]. Excessive focus on local solutions is the biggest deficiency for ANN especially in the training phase. The hybrid algorithms that are created by completing the deficiencies of an algorithm with a heuristic algorithm make it possible to obtain high performance results although working slowly and [12-18]. In these studies, hybrid approaches have been used to optimize gene selection on microarray data. In this study, In order to complete the deficiencies of ANN, a hybrid approach was developed. ANN was supported by the fire fly algorithm which is an optimization algorithm. With this particle 
swarm based algorithm inspired by the interaction of fireflies with each other, it is aimed to prevent artificial neural networks from being attached to local solutions in the training phase.

The study divided into four sections is made up of like this scheme: In the first section, the advantages of heuristic and hybrid approaches as well as their use in the literature are provided. In the second section the practice where artificial neural networks that are optimized by firefly that is prepared for cancer diagnosis and its working principle is explained. In the next section, the advantageous and disadvantageous sides of algorithms are discussed by comparing the findings obtained from the method deployed and from other algorithms. In the last section, results are provided and references to further studies are given.

\section{MATERIAL METHOD}

Artificial Neural Networks are systems made up of artificial nerve cells that imitate problem solving skills of biological nerve cells by communication and that only living things possess [19]. These networks discover the connections among attributions inside the samples and can make it possible to classify a sample that they have never encountered before. The discovery process of connections among previous samples is called the training of artificial neural networks. The classification plays an important role for the accuracy of results. However, when an appropriate solution is found for network classification during the process, it can be possible to focus on this solution. At this point, the artificial neural network algorithm is optimized with the help of firefly algorithm, which is a powerful and fast optimization algorithm, in order to decrease this sticking possibility.

\subsection{Artificial Neural Networks}

A simple working principle of artificial neural network is shown in Figure 1.

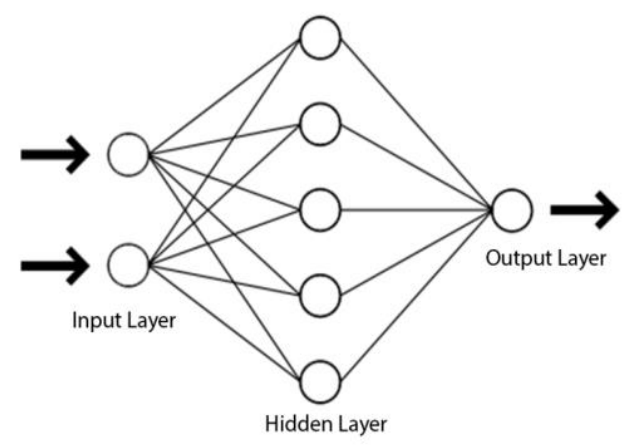

Figure 1. Structure of a simple artificial neural network

Input layer is the part where the attributions for each sample are given to the system (Equation 1). These data are transferred from input layer to each nerve in the next layer by being multiplied by weights that were created randomly in the beginning (Equation 2). The output of each nerve in the mid layer is obtained by passing the data coming from the previous layer through adding and transfer functions (Equation 3). In the output layer, an estimation process is carried out by repeating the processes in the previous layer because ofthe total of the data coming from the mid layer. As a result of this estimation, Mean Squared Error (MSE) is calculated between the real output of samples and the estimated output (Equation). The training would be completed according to this error through error back propagation method by updating the each weight on network (Equation 4). By repeating this process, the least possible error, namely the optimum value, is tried to be obtained

$C_{i}^{G}=A_{i}$,

$N e t_{j}^{A}=\sum_{k=1}^{n} A_{k j}^{G A} \cdot C_{k}^{G}$, 
$C_{j}^{A}=\frac{1}{\left.1+e^{-(} \operatorname{Net}_{j}^{A}+\beta_{j}^{a}\right)}$

$M S E=\frac{1}{2} \sum_{m}\left(B_{m}-C_{m}^{C}\right)^{2}$.

In Equation (1), $A_{i}$ stands for each attribution of the samples that will be given to the network where $C_{i}^{G}$ stands for the output of each nerve in the input layer. In Equation (2), $N e t_{j}^{A}$ stands for the total value of each nerve calculated in the mid layer where $A_{k j}^{G A}$ is the expression of each weight between itself and the previous layer. The $C_{j}^{A}$ used in Equation (3) is the value of each nerve output and $\beta_{j}^{a}$ is the correction value chosen as a constant - and also known as Bias - that is used in many algorithms such as artificial neural networks. In Equation (4), MSE is Mean Squared Error, $B_{m}$ is expected output, $C_{m}^{C}$ is the realized output.

\subsection{Fire Fly Algorithm and The Developed Hybrid Algorithm}

The fire fly algorithm is an optimization algorithm that is based upon the interaction of fireflies, just like other sensational algorithm based on nature [20]. In the study, Yang explained these terms:

- All the fire flies are sex free. Therefore, each fire fly can be attracted by another one,

- The attractiveness of fire flies is in parallel with their brightness. Therefore, fire flies that are brighter attract the ones that are less bright. If a brighter fire fly cannot be found, then the fire fly behaves randomly,

- The brightness of the fire fly is identified among the purpose function gap.

Setting off from these explanations, in order to optimize the artificial neural network, the two algorithms is combined like this; Each fire fly is a possible solution within the solution set as an individual that represents the weight of the artificial neural networks. The purpose here is to enable the artificial neural networks to operate with the least MSE rate by determining the most appropriate weights. In this case, the purpose function is known as the minimisation of artificial neural errors. The flow diagram of the created system is shown in Figure 2. 


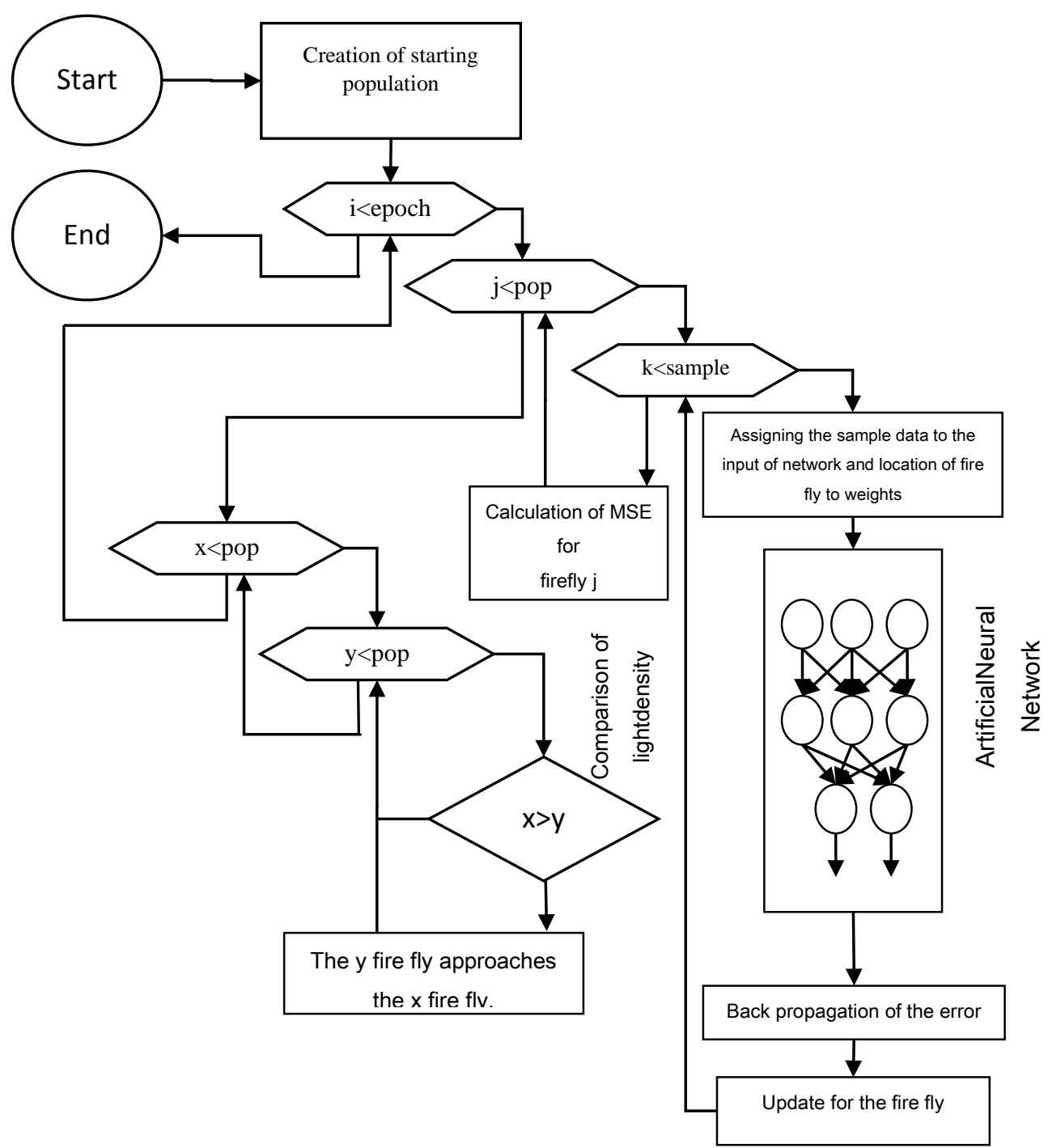

Figure 2. The developed artificial neural network and the flow diagram of fire fly hybrid algorithm

In the algorithm, a starting population is created initially as shown in Figure 2. While creating the starting population, the number of fire flies in the population have to be determined. That less number of individuals are selected may lead to the fact that algorithm works fast but it will also lead to the fact that the algorithm scans the solution sets with less numbers of fire flies. This will decrease the possibility of finding the most appropriate solution. Having more numbers of fire flies will increase the possibility of finding the most appropriate solution. Having excessive numbers will increase the calculation costs and can create a work load today's computers cannot overcome. Due to the fact that the co-efficient required for providing the attractiveness light absorption and randomness that are used together with the fire fly number in the algorithm does not have a definite starting value, the value needs to be determined by trial and error. After the parameters are determined, the algorithm give the fire flies a starting location.

The i loop enable the algorithm to be repeated as much as the chosen step number. Every time this loop repeats itself, it triggers the $\mathrm{j}$ loop for each fire fly, and the $\mathrm{j}$ loop triggers $\mathrm{k}$ loop for each sample in the data set. By this means, it can be used as the weight of artificial neural network for each sample in each fire fly data set. The completion of artificial neural network is changed as a result of the output and error value it obtained while the location of the fire flies is changed by the back propagation algorithm.

After the completion of the $\mathrm{j}$ loop, the $\mathrm{x}$ and $\mathrm{y}$ loop are run in a concentric way in order to provide the interaction of the fire flies among each other. The $\mathrm{x}$ and $\mathrm{y}$ loops are run for each fire fly. Thus, each fire fly can be compared to the light density of another one. The brightness of the fireflies is indicated in an inversely proportional way with the purpose function. Due to the fact that the purpose function within the 
algorithm is to minimize the error of artificial nerve network, the MSE value obtained from each fire fly is calculated as the brightness of the fire fly in an inversely proportional way as shown in Equation (5)

$I_{i}=\frac{1}{\left(1+M S E_{i}\right)}$

Upon the comparison of the brightness values, the distance between two fire fly is calculated as shown in Equation (6)

$r_{x y}=\sqrt{\sum_{k}^{n}\left(x_{k}-y_{k}\right)^{2}}$.

The $\mathrm{x}$ and $\mathrm{y}$ shown in the equation indicate the distance between fire flies, $x_{k}$ indicates the $\mathrm{k}$ attribution of the fire fly $x_{k}, y_{k}$ shows the $\mathrm{k}$ attribution of the fire fly $\mathrm{y}$. The $\mathrm{n}$ variable indicates the number of attributes that each fire fly possesses. By replacing square of the distance calculated in Equation (6) with the movement from fire fly with less light density towards the bright fire fly, changes in each attribution of the fire fly is calculated as in Equation (7)

$x_{i}=x_{i}+\beta e^{-\gamma r_{x y}^{2}}\left(x_{i}-y_{i}\right) \alpha$.

$\beta$ indicates the attraction status between two fire flies and $\gamma$ indicates the light absorption value. $\alpha$ is a variable added in order to provide the randomness that can take random values in limited gaps.

After the termination of i loop, the fire fly with the least MSE is chosen for the test process.

\subsection{The Developed Interface}

Deciding on the parameters that are required for heuristic algorithms to run is a difficult process. That these algorithms do not work linearly makes the estimation of reaction to the parameter difficult. Therefore, an easy-to-use and useful interface that user can use to alternate the parameters of artificial neural network, fire fly algorithm and the genetic algorithm that is used for comparison and also that user can observe the effect of the changes on output was prepared (Figure 3).

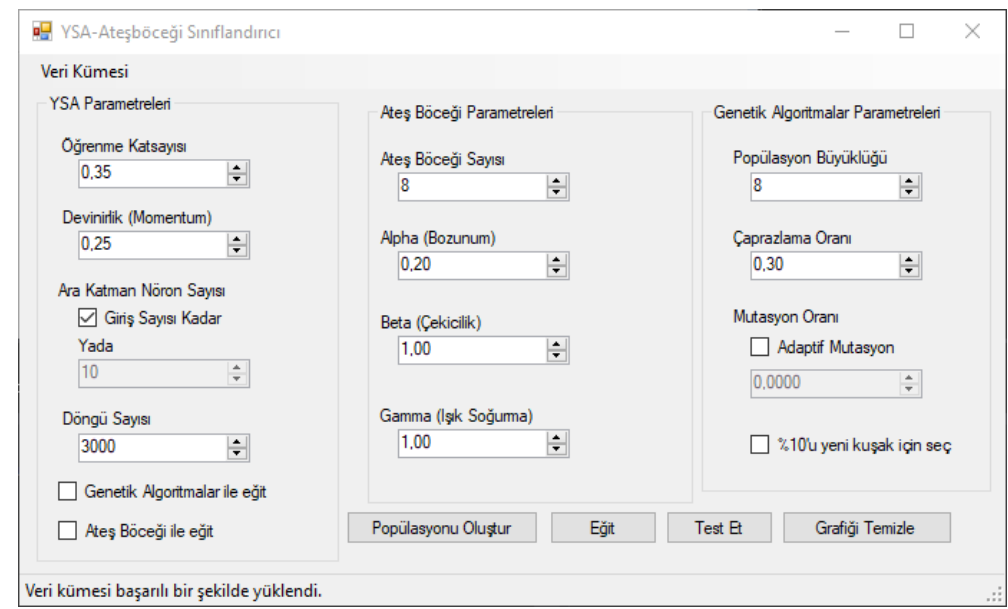

Figure 3. The developed Interface

The interface was developed in a flexible structure where training and test data sets can be added to the developed algorithm so that users can make trials through their own data sets. It supports most of today's file extensions among which .CVS, .TXT, and .XML can be included.

After loading the training and test data sets and after setting the parameters of artificial neural networks, not only can users choose the desired optimisation technique but also they can train the artificial neural 
network as they wish. During the training period, the mse/epoch graph in each step can be observed in real time.

\section{THE RESEARCH FINDINGS AND DISCUSSION}

The algorithm - prepared by Pomeroy, Tamayo, Gaasenbeek, Sturla, and Angelo, 2002 [21]- for which a data set out of the micro array data taken from patients with central nervous system tumour was chosen to compare with the developed algorithm and other heuristic approaches used in the literature. There are 60 samples in the data set and each sample has attributions that represent 7129 genes. Data set has two classes (Class 1 - survives : 39, Class 2 - worsen disease : 21 ) A new data set was obtained carried to a different dimension with 15 attributions by applying Basic Component analysis in the data set in order to decrease the calculation cost and increase distinctiveness in the data set. After allocating $80 \%$ for the training and $20 \%$ for the test, plain artificial neural systems are separately compared to the results obtained from the artificial neural system optimised with fire fly and from the artificial neural system optimised with genetic algorithm. The mse/epoch graph in training phases are provided in Figure 4.

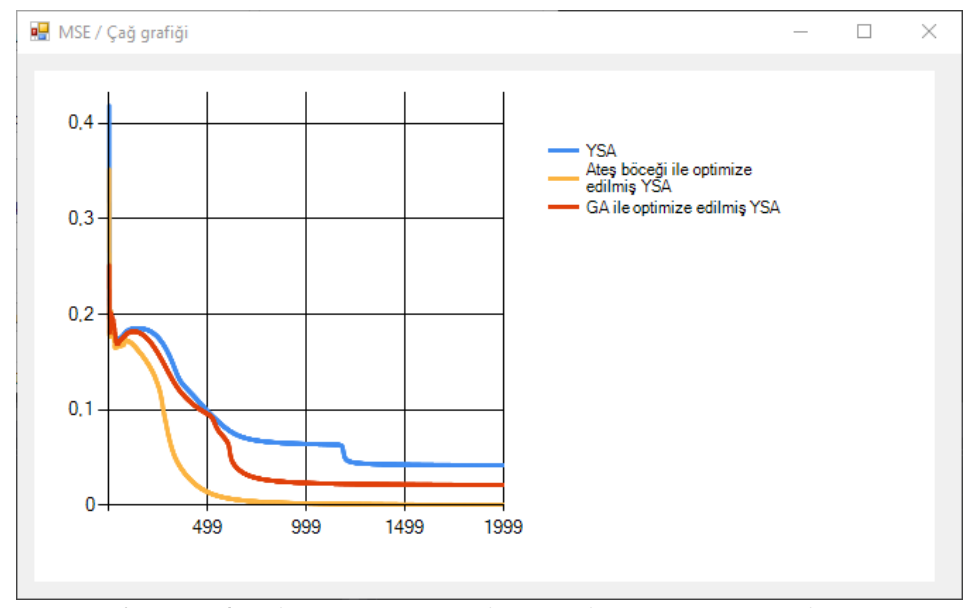

Figure 4. The MSE/Epoch graph in training phases

It is seen that the network halts in various local minimums when the curve obtained from the training with artificial neural networks only is studied. Eventually, it is understood that the error does not show any declining trend by accepting the local minimum obtained at around $1200^{\text {th }}$ epochs and by terminating the training process. When the curve of artificial neural network optimised by genetic algorithm is studied, it is seen that errors are instantly noticed and a faster training is obtained. Finally, when the curve of artificial neural network that was trained by fire fly algorithm is compared to the others, it is observed that both it passes the minimum levels fast and obtains a more optimum value by reaching the lowest MSE value.

The performance values of 3 algorithms training of which was completed as well as of those results of which are obtained when they are tested with KNN and SVM for classification in the literature are provided in Table 1. 
Table 1. The performance values obtained from the algorithm as a result of test processes.

\begin{tabular}{|c|c|c|c|c|c|c|c|c|}
\hline \multirow[t]{2}{*}{ Algorithm } & \multirow[t]{2}{*}{ Clearance } & \multirow[t]{2}{*}{ Sensitivity } & \multirow[t]{2}{*}{ Accuracy } & \multirow[t]{2}{*}{$\overline{\text { AUC }}$} & \multirow[t]{2}{*}{ Regression } & \multicolumn{3}{|c|}{ Time lapse (sn) } \\
\hline & & & & & & Training & Test & Total \\
\hline ANN & 0.75 & 0.625 & 0.66 & 0.53 & 0.333 & 11.18 & 22.5 & 33.68 \\
\hline $\begin{array}{l}\text { ANN opt. with } \\
\text { GA }\end{array}$ & 0.80 & 0.71 & 0.75 & 0.73 & 0.507 & 72.38 & 22.5 & 94.88 \\
\hline $\begin{array}{l}\text { ANN opt. with } \\
\text { FFA }\end{array}$ & 0.80 & 0.71 & 0.75 & 0.73 & 0.507 & 35.31 & 22.5 & 57.81 \\
\hline KNN & 0.66 & 0.66 & 0.66 & 0.65 & 0.353 & --- & 0.01 & 0.01 \\
\hline SVM & 0.2 & 0,857 & 0.58 & 0.53 & 0.333 & --- & 0.01 & 0.01 \\
\hline
\end{tabular}

When the performance values concerning the algorithms are studied, it is seen that the artificial neural network optimized with Fire fly returns more successful results than those powerful algorithms such as KNN, SVM and ANN. Despite the fact that the developed algorithm returns the most appropriate MSE value, it is seen that it provides the same performance with the artificial neural network with genetic algorithm. That the number of samples in the selected data set is less but has more attributions shows that it shows a resistance against the rise of the success rate. It is observed that optimisation algorithms increase the time elapsed for the training but also that fire fly algorithm runs faster than genetic algorithm.

The area under the curve (AUC) is one of the measurement methods used to perform performance measurements in classification procedures. This method scores the classification ability of algorithms by looking at the discrimination rate between classes. The method scales the algorithms between 0 and 1 . The values approaching 1 indicate a better separation between survive and worsen disease classes. When the obtained AUC performance values are examined, it is observed that hybrid approaches achieve better results. When the obtained AUC performance values are examined, it is observed that hybrid approaches better distinguish the classes.

\section{RESULTS}

As a result of the study carried out, an application was developed where users can do some training using different parameters for artificial neural network optimised by fire fly, which is a hybrid approach; where users can do testing; and where users can observe the outputs on real-time graphics. An easy-to-use and plain working environment where users can add their own data sets was created.

During the training phase, the prepared hybrid algorithm could reach a more optimum value that has a lower error rate when compared to artificial neural networks optimised with ANN and genetic algorithm. During the test phase, it obtained a better performance value than ANN. The fact that the data set is complex led to the fact that similar performance values are obtained with the artificial neural network optimised with genetic algorithm, which is a hybrid approach, because it poses an obstacle in the increase of the classification success. Nevertheless, the learning skills of hybrid approaches as well as their ability to solve problems without requiring initial knowledge promise hope for future studies

In the future studies, it is planned to design an interface where more parameters can be added and changed, where sections can be differently assigned to either hybrid or plain structures; and where similar numerous developments can be done. It is also planned to try the training phase of the developed algorithm on more powerful computers during longer epochs and therefore to observe the behaviour in the long term.

\section{CONFLICTS OF INTEREST}

No conflict of interest was declared by the authors. 


\section{REFERENCES}

[1] Cooper, C.S., "Applications of microarray technology in breast cancer research", Breast Cancer Research, 3(3):158, (2001).

[2] Lv, J., Peng, Q., Chen, X., Sun, Z., “A Multi-objective Heuristic Algorithm for Gene Expression Microarray Data Classification”, Expert Systems with Applications, 59:13-19, (2016).

[3] Fakoor, R., Ladhak, F., Nazi, A., Huber, M., "Using Deep Learning to Enhance Cancer Diagnosis and Classification", Proceedings of the 30th International Conference on Machine Learning, Atlanta, Georgia (2013).

[4] Danaee, P., Ghaeini, R., Hendrix, D. A., "A Deep Learning Approach for Cancer Detection and Relevant Gene Identification", In Pacific Symposium on Biocomputing, Pacific Symposium on Biocomputing, Big Island, Hawaii, 22:219-227, (2017).

[5] Yu, Z., Chen, H., You, J., Liu, J., Wong, HS., Han, G., Li, L., "Adaptive fuzzy consensus clustering framework for clustering analysis of cancer data", IEEE/ACM Transactions on Computational Biology and Bioinformatics, 12(4):887-901, (2015).

[6] Borkowska, E., M., Kruk, A., Jedrzejczyk, A., Rozniecki, M., Jablonowski, Z., Traczyk, M., Constantinou, M., Banaszkiewicz, M., Pietrusinski, M., Sosnowski, M., et al., "Molecular Subtyping of Bladder Cancer using K Ohonen Self-organizing Maps" Cancer Medicine, 3(5):12251234, (2014).

[7] Leung, M., M., K., Delong, A., Alipanahi, B., Frey, B., J. ,"Machine Learning in Genomic Medicine: A Review of Computational Problems and Data Sets", Proceedings of The IEEE, 104(1):176-197, (2016).

[8] Petalidis, L. P., Oulas, A., Backlund, M., Wayland, M. T., Liu, L., Plant, K., et al., "Improved grading and survival prediction of human astrocytic brain tumors by artificial neural network analysis of gene expression microarray data", Mol Cancer Ther, 7(5):1013-1024,(2008).

[9] Ellis, M., Davis, N., Coop, A., Liu, M., Schumaker, L., Lee, R. Y., et al., "Development and Validation of a Method for Using Breast Core Needle Biopsies for Gene Expression Microarray Analyses", Clinical Cancer Research, 8:1155-1166, (2002).

[10] Peterson, L. E., \& Coleman, M. A., "Machine learning-based receiver operating characteristic (ROC) curves for crisp and fuzzy classification of DNA microarrays in cancer research", International Journal of Approximate Reasoning, 47(1):17-36, (2008).

[11] Bicciato, S., Pandin, M., Didone, G., \& Bello, C. D. ,"Pattern identification and classification in gene expression data using an autoassociative neural network model", Biotechology and Bioengineering, 81:594-606, (2002).

[12] Shukla, A., K., Singh, P., Vardhan, M., "A Two-stage Gene Selection Method for Biomarker Discovery from Microarray Data for Cancer Classification", Chemometrics and Intelligent Laboratory Systems, 183:47-58, (2018).

[13] Tabakhi, S., Najafi, A., Ranjbar, R., Moradi, P., "Gene Selection for Microarray Data Classification using a Novel Ant Colony Optimization", Neurocomputing, 168:1024-1036, (2015).

[14] Rani, R. R., Ramyachitra, D., "Microarray Cancer Gene Feature Selection Using Spider Monkey Optimization Algorithm and Cancer Classification using SVM", Procedia Computer Science, 
143:108-116, (2018).

[15] Peng, S., Xu, Q., Ling, X. B., Peng, X., Du, W., Chen, L., "Molecular classification of cancer types from microarray data using combination of genetic algorithms and support vector machines", FEBS Letters, 555(2):358-362, (2003).

[16] Li, L., Jiang, W., Li, X., Moser, K. L., Guo, Z., Du, L., et al., “A robust hybrid between genetic algorithm and support vector machine for extracting an optimal feature gene subset", Genomics, 85(1):16-23, (2005).

[17] Rao, A. C., Somayajulu, D., Banka, H., \& Chaturverdi, R., "Outliner Detection in Micoarray Data Using Hybrid Evolutionary Algorithm”, Proceida Technology, 6:291-298, (2012).

[18] Kumar, P. G., Victoire, T. A., Renukadevi, P., \& Devaraj, D., "Design of fuzzy expert system for microarray data classification using a novel Genetic Swarm Algorithm", Expert Systems with Applications, 39(2):1811-1821, (2012).

[19] Blanton, H. ,"An introduction to neural networks for technicians, engineers and other non PhDs.”, Proceedings of the 1997 Artificial Neural Networks in Engineering Conference, St. Louis, (1997).

[20] Yang, X. S. Nature-Inspired Metaheuristic Algorithms. UK: Luniver Press, (2008).

[21] Pomeroy, S. L., Tamayo, P., Gaasenbeek, M., Sturla, L. M., Angelo, M., "Prediction of central nervous system embryonal tumor outcome based on gene expression", Letter to Nature, 436-442, (2002). 